\title{
ON FOUR-DIMENSIONAL $h$-COBORDISM
}

\author{
SLAWOMOR KWASIK
}

\begin{abstract}
The influence of the Poincare Conjecture on the following problem of $\mathrm{M}$. Cohen is examined.

Problem. Does there exist a four-dimensional $h$-cobordism with nontrivial Whitehead torsion?

It is proved (assuming the Poincarè Conjecture) that none of the following groups may be the fundamental group of a 4-dimensional $h$-corbordism with nontrivial Whitehead torsion: generalized quaternion groups, cyclic groups $Z_{8}, Z_{12}$, the binary octahedral group, the binary tetrahedral group.
\end{abstract}

There is a well-known procedure for constructing $h$-cobordisms with nontrivial Whitehead torsion. In fact, given a closed manifold $M_{0}^{n-1}, n-1 \geqslant 4$, then there is an $h$-cobordism $\left(W^{n}, M_{0}^{n-1}, M_{1}^{n-1}\right)$ which realizes any prescribed torsion element $\tau \in \mathrm{Wh}\left(Z\left[\pi_{1}\left(M_{0}^{n-1}\right)\right]\right)$ (see [4, 10]). The cases $n-1=2$ and $n-1=1$ are trivial because $\operatorname{Wh}\left(Z\left[\pi_{1}\left(M^{2}\right)\right]\right)=\mathrm{Wh}\left(Z\left[\pi_{1}\left(M^{1}\right)\right]\right)=0$ (see [12, 4]). Almost nothing is known for $n-1=3$, and the following intriguing problem was posed by M. Cohen (see [1]):

Problem. Does there exist a four-dimensional $h$-cobordism with nontrivial Whitehead torsion?

Note that if $\pi_{1}\left(M^{3}\right)$ is infinite then $M^{3}$ is a $K\left(\pi_{1}\left(M^{3}\right) ; 1\right)$-manifold (or $M^{3}=S^{1}$ $\times S^{2}$ ) and there is a well-known conjecture (supported by the case of sufficiently large manifolds (see [12]) and Seifert manifolds (see [6])) that $\mathrm{Wh}\left(Z\left[\pi_{1}\left(M^{3}\right)\right]\right)=0$. Therefore it seems to be reasonable to restrict our considerations to the case where $\pi_{1}\left(M^{3}\right)$ is a finite group. Every finite 3-manifold group can act freely on $S^{3}$ (maybe on fake $S^{3}$ ) and all such are classified (see, for example, [3]). The purpose of this note is to show the influence of the Poincare Conjecture on the above problem. Modifying Milnor's argument from [4] we prove the following

THEOREM. If the Poincare Conjecture is true then none of the following groups may be the fundamental group of a four-dimensional h-cobordism with nontrivial Whitehead torsion:

(a) generalized quaternion groups,

(b) cyclic groups $Z_{8}$ and $Z_{12}$,

(c) the binary octahedral group $O(48)$,

(d) the binary tetrahedral group $T(24)$.

Received by the editors April 9, 1985.

1980 Mathematics Subject Classification. Primary 57R80; Secondary 57S17.

C1986 American Mathematical Society $0002-9939 / 86 \$ 1.00+\$ .25$ per page 
If in addition every free action of a finite group on $S^{3}$ is equivalent to the orthogonal one, then the following groups are excluded:

(a) all quaternion groups $Q(4 n)$,

(b) all cyclic groups $Z_{n}$,

(c) the groups $O(48)$ and $T(24)$,

(d) the binary icosahedral group I(120).

Proof. First we show how the Poincare Conjecture eliminates the generalized quaternion groups. From the proof of this case it becomes clear that the remaining groups are eliminated too. Let $M_{0}$ be a 3-dimensional closed manifold with $\pi_{1}\left(M_{0}\right)=Q 2^{n}, n \geqslant 3$, and let $\left(W ; M_{0}, M_{1}\right)$ be an $h$-cobordism. We show that $\tau\left(W ; M_{0}\right)=0$. Consider the universal covering $\tilde{W}$ of $W$. The boundary $\partial \tilde{W}$ of $\tilde{W}$ is a disjoint union of $\Sigma_{1}^{3}$ and $\Sigma_{2}^{3}$, where $\Sigma_{1,2}^{3}$ is a homotopy 3-sphere; by our assumption $\Sigma_{1.2}^{3}=S^{3}$. Therefore we have an $h$-cobordism $\left(\tilde{W} ; S^{3}, S^{3}\right)$ with a free action of $Q 2^{n}$. It is proved in [11] that every free action of $Q 2^{n}$ on $S^{3}$ is equivalent to the orthogonal one. This implies that both manifolds $M_{0}=S^{3} / Q 2^{n}$ and $M_{1}=$ $S^{3} / Q 2^{n}$ are Seifert manifolds and hence are diffeomorphic (see [8]) and, in fact, are isometric. To simplify our notation from now on we put $Q 2^{n}=G$. Let $i: Z[G] \rightarrow$ $Q[G]$ be the natural inclusion. It induces the homomorphism $i_{*}: K_{1}(Z[G]) \rightarrow$ $K_{1}(Q[G])$; we denote the kernel of $i_{*}$ by $S K_{1}(Z[G])$ and the image by $K^{\prime}(Z[G])$. Note that $K_{1}(Q[G])=K_{1}(Q) \oplus K_{1}\left(Q R_{G}\right)$ where $Q R_{G}$ is defined by $Q R_{G}=$ $Q[G] /(\Sigma)$ and $\Sigma$ denotes the sum of elements of $G$. Because $G$ acts trivially on $H_{*}\left(S^{3} ; Q\right)$, the Reidemeister torsion $\Delta(\cdot) \in \bar{K}_{1}\left(Q R_{G}\right) / \operatorname{Im} G$ is defined (see [4]). One has (see [4]) the following formula (after the identification of fundamental groups):

$$
\Delta(W)=\Delta\left(W ; M_{0}\right) \cdot \Delta\left(M_{0}\right)=\Delta\left(W, M_{1}\right) \cdot \Delta\left(M_{1}\right) .
$$

It was proved in [7] that $S K_{1}(Z[G])=0$ which implies (see [13]) that $\mathrm{Wh}(Z[G])$ can be identified with

$$
\mathrm{Wh}^{\prime}(Z[G]):=\mathrm{Wh}(Z[G]) / S K_{1}(Z[G]) .
$$

Therefore, $\mathrm{Wh}(Z[G])$ is a free abelian group and the standard involution *: $\mathrm{Wh}(Z[G]) \rightarrow \mathrm{Wh}(Z[G])$ is trivial (see [13]). Because $\left(W ; M_{0}, M_{1}\right)$ is an $h$-cobordism then the Whitehead torsion $\tau\left(W, M_{i}\right) \in \mathrm{Wh}(Z[G]), i=1,2$, is defined. It is not difficult to see that $\Delta\left(W ; M_{i}\right)$ is the image of $\tau\left(W, M_{i}\right), i=1,2$, under the natural identification.

For, consider the following commutative diagram:

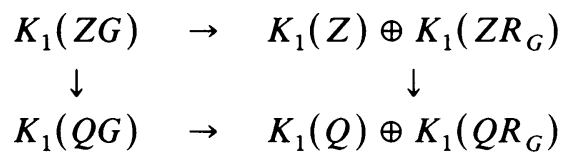

Since $\mathrm{Wh}(Z G)=\bar{K}_{1}(Z G) /$ torsion, the commutativity of the above diagram, together with the fact that $\bar{K}_{1}(Z) /$ torsion $=0$, shows that $\mathrm{Wh}(Z G)$ injects into $\bar{K}_{1}\left(Q R_{G}\right)$. Therefore, by an argument analogous to that in [4] one concludes that $2 \tau\left(W, M_{0}\right)=0$ and, hence, $\tau\left(W ; M_{0}\right)=0$ because $\mathrm{Wh}(Z[G])$ is torsion free. This 
completes the case $G=Q 2^{n}$. For $G=O(48)$ and $G=T(24)$, hyperelementary induction shows tht $S K_{1}(Z[G])=0$. Because the free actions of these groups on $S^{3}$ are equivalent to orthogonal actions (see [11]), then the desired result follows. The case $G=Z_{8}$ and $G=Z_{12}$ follows from $[4$ and 9,11$]$. Note that here the Atiyah-Bott result is needed. Now if there are only orthogonal free actions on $S^{3}$, then for every group $G$ in the statement of the theorem $S K_{1}(Z[G])=0$ and this is all that is needed.

REMARK 1. It is quite probable that using this method one can eliminate some other groups too, but definitely not all. For example, $S K_{1}(Z[G])$ is in general nontrivial (see [7]) for groups given by $G=Z_{p} \times Q 2^{n}$, $p$-prime.

REMARK 2. As it was mentioned at the beginning of this note for every closed manifold $M^{n}, n \neq 3$, and every $\tau \in \mathrm{Wh}\left(Z\left[\pi_{1}\left(M^{n}\right)\right]\right)$ there exists an $h$-cobordism which realizes $\tau$. From our theorem one can conclude that this is no longer the case when $n=3$, at least if the Poincaré Conjecture is true. Namely, we have the following

COROllary. If the Poincare Conjecture is true then there are infinitely many examples which show that the four-dimensional version of the realization theorem for Whitehead torsion is false.

Proof. It is enough to show that $\mathrm{Wh}\left(Z\left[Q 2^{n}\right]\right) \neq 0$ for $n \geqslant 4$. This is true and implicitly it is contained for example in [14]. Note, that $\mathrm{Wh}(Z[Q 8])=0$.

REMARK 3. The above Corollary was also proved in [2] using slightly different methods.

I would like to thank the referee for his corrections and suggestions on improving the exposition.

\section{REFERENCES}

1. R. C. Kirby, Problems in low dimensional manifold theory, Proc. Sympos. Pure Math., Vol. 32, Amer. Math. Soc., Providence, R. I., 1978, pp. 273-312.

2. S. Kwasik, On realization of Whitehead torsion, Math. Proc. Cambridge Philos. Soc. 98 (1985), 271-273.

3. R. Lee and C. B. Thomas, Free actions by finite groups on $S^{3}$, Bull. Amer. Math. Soc. 79 (1973), 211-215.

4. J. Milnor, Whitehead torsion, Bull. Amer. Math. Soc. 72 (1966), 358-426.

5. Introduction to algebraic K-theory, Ann. of Math. Studies, No. 72, Princeton Univ. Press, Princeton, N. J., 1971

6. S. P. Plotnik, Vanishing of Whitehead groups for Seifert manifolds with infinite fundamental group, Comment. Math. Helv. 55 (1980), 654-667.

7. R. Oliver, $S K_{1}$ for finite group rings. III, Algebraic K-theory (Evanston 1980), Lecture Notes in Math., vol. 854, Springer-Verlag, Berlin and New York, 1981, pp. 299-337.

8. P. Orlik, Seifert manifolds, Lecture Notes in Math., vol. 291, Springer-Verlag, Berlin and New York, 1972.

9. G. Ritter, Free actions of $Z_{8}$ on $S^{3}$, Trans. Amer. Math. Soc. 181 (1973), 195-212.

10. C. P. Rourke and B. J. Sanderson, Introduction to piecewise linear topologv, Ergebnisse der Math. und ihrer Grenzgebiete, Band 69, Springer-Verlag, Berlin and New York, 1972.

11. J. H. Rubinstein, Free actions of some finite groups on $S^{3}$. I, Math. Ann. 240 (1979), 165-175.

12. F. Waldhausen, Algebraic $K$-theory of generalized free products, Ann. of Math. 108 (1978), 135-256.

13. C. T. C. Wall, Norms of units in group rings, Proc. London Math. Soc. (3) 29 (1974), 593-632.

14. , Periodic projective resolutions, Proc. London Math. Soc. (3) 39 (1979), 509-553.

Department of Mathematics, Purdue University, West LafayetTe. Indiana 47907 